Revue internationale P.M.E.

Économie et gestion de la petite et moyenne entreprise

Revue

internationale

PME

\title{
Pérennisation d'une PME : la spécificité du processus stratégique
}

\section{Sophie Mignon}

Volume 15, numéro 2, 2002

URI : https://id.erudit.org/iderudit/1008808ar

DOI : https://doi.org/10.7202/1008808ar

Aller au sommaire du numéro

Éditeur(s)

Presses de l’Université du Québec

ISSN

0776-5436 (imprimé)

1918-9699 (numérique)

Découvrir la revue

Citer cette note

Mignon, S. (2002). Pérennisation d’une PME : la spécificité du processus stratégique. Revue internationale P.M.E., 15(2), 93-118.

https://doi.org/10.7202/1008808ar
Résumé de l'article

Cet article décrit le processus de formation des stratégies d'une entreprise pérenne sur un plan organisationnel. Le processus de variation, sélection et rétention des initiatives stratégiques au niveau intra-organisationnel est analysé à partir d'une étude de cas. On y met en évidence l'existence de filtres (continuité stratégique, priorité accordée au client, perpétuation d'un savoir-faire, valeurs morales, éthique, responsabilité sociale, etc.) qui ont permis de " contextualiser » les initiatives stratégiques. Ces éléments de contexte interne constituent ainsi une aide à la décision stratégique en permettant l'abandon de certaines options et la rétention de stratégies plus pertinentes en termes de pérennité. Les différences entre le processus d'adaptation et celui de proaction concourant à la pérennité sont également soulignées. 


\title{
Pérennisation d'une PME: la spécificité du processus stratégique
}

Sophie MIGNON

Université de Montpellier II

\section{MOTS CLÉS}

\section{PME - Processus stratégique - Continuité - Sélection Adaptation - Proaction - Pérennité}

\section{RÉSUMÉ}

Cet article décrit le processus de formation des stratégies d'une entreprise pérenne sur un plan organisationnel. Le processus de variation, sélection et rétention des initiatives stratégiques au niveau intra-organisationnel est analysé à partir d'une étude de cas. On y met en évidence l'existence de filtres (continuité stratégique, priorité accordée au client, perpétuation d'un savoir-faire, valeurs morales, éthique, responsabilité sociale, etc.) qui ont permis de "contextualiser" les initiatives stratégiques. Ces éléments de contexte interne constituent ainsi une aide à la décision stratégique en permettant l'abandon de certaines options et la rétention de stratégies plus pertinentes en termes de pérennité. Les différences entre le processus d'adaptation et celui de proaction concourant à la pérennité sont également soulignées.

\begin{abstract}
This article highlights the strategy elaboration process of a sustainable firm from an organizational point of view. The process of diversifying, selecting and retaining strategic initiatives at an intra-organizational level is analysed on the basis of a case
\end{abstract}

\section{L'AUTEURE}

SOPHIE MIGNON est maître de conférences à l'Université de Montpellier II. Ancienne élève de I'ENS Cachan, agrégée d'économie et gestion et docteure en sciences de gestion, ses travaux de recherche et publications portent sur les thèmes de l'histoire des entreprises, du processus de formation des stratégies, de la pérennité des petites et moyennes entreprises et du management des connaissances. Adresse : Université de Montpellier II (IUT), 99, avenue d'Occitanie, 34296 Montpellier Cedex 5, France. 
study. Attention will also focus on the role of filters i.e. strategic continuity, priority granted to clients, perpetuation of know-how, moral values, ethics, social responsibility, in "contextualizing" strategic initiatives. These elements, which are part of the internal context, thus contribute to the strategic decision-making process, allowing for certain options to be abandoned in favour of more appropriate strategies in terms of sustainability. Emphasis is also placed on the differences between the process of adaptation and that of pro-action leading to sustainability.

\section{RESUMEN}

En este articulo se trata del proceso de formación de las estrategias de una empresa perenne en el plano de la organización. Se analiza el proceso de variación, selección y retención de las iniciativas estratégicas al nivel intra-organizacional a partir de un estudio de casos. Se pone de relieve la existencia de filtros (continuidad estratégica, prioridad al cliente, perpetuación de know-how, valores morales, ética, responsabilidad social...) que permitieron " contextualizar » las iniciativas estratégicas. Estos elementos de contexto interno constituyen asi una ayuda a la decisión estratégica permitiendo abandonar algunas opciones y elegir estrategias màs pertinentes respecto a la perennidad. Se subrayan también las diferencias entre el proceso de adaptación y el de pro-acción que contribuyen a la perennidad.

\section{ZUSAMMENFASSUNG}

Dieser Artikel hebt den Prozess der Strategiebildung einer fortdauernden Unternehmung hervor, aufbauend auf einem organisatorischen Plan. Der Prozess der Variation, der Auswahl und das Festhalten an den strategischen Initiativen auf dem inner-organisatorischen Niveau wurde mit einer Fallstudie analysiert. Die Existenz von Filtern wurde klargestellt (strategische Dauer, zugewiesene Priorität an die Kunden, Fortbestand der Umsetzung, moralische Werte, Ethik, soziale Verantwortung, etc..), die es erlaubt haben, die strategischen Initiativen in den Kontext zu setzen. Diese Elemente des internen Kontextes bilden also eine Hilfe für strategische Entscheide und erlauben die Vernachlässigung von gewissen Optionen und das Festhalten an zutreffenden Strategien für den Unternehmensfortbestand. Die Unterschiede zwischen dem Anpassungsprozess und denjenigen der aktiven Konkurrenzstrategien sind für den Fortbestand ebenso unterstrichen worden.

\section{Introduction}

Lorsque Babyliss, PME du Nord, fut vendue il y a quelques années au groupe Conair, l'objectif affiché par le dirigeant-propriétaire prit la forme suivante: «C'était pour moi la meilleure façon d'assurer la pérennité de mon entreprise. Je n'aurai pas de successeur. J'ai décidé de passer la main à un partenaire que j'aurai choisi. Conair a la même histoire que nous. N'ayant aucune implantation en Europe, il ne va pas mettre en péril l'organisation. »(Le Monde, 23 décembre 1995)

Revue internationale P.M.E., vol. 15, nº 2, 2002 
Si le maintien de l'organisation constitue une perception possible de la pérennité d'une entreprise, les formes que revêt cette dernière sont multiples. Plus précisément, il est possible de distinguer deux grandes familles de pérennité (Mignon, 1998, 2000) ${ }^{1}$ :

- la pérennité du pouvoir: celle-ci recouvre deux types de pérennité. La pérennité du contrôle est assurée lorsque le capital reste entre les mains du même groupe d'actionnaires (en général un individu ou une famille) et la pérennité de direction est réalisée lorsque les dirigeants de l'entreprise sont issus de ce même groupe ;

- la pérennité du projet : celle-ci recouvre, également, deux types de pérennité. La pérennité des activités est assurée lorsque l'activité principale de l'entreprise est maintenue, en dépit, par exemple, d'une disparition de cette dernière en tant qu'entité autonome. La pérennité organisationnelle est préservée lorsque l'entreprise a su, au cours de son histoire, résister à l'épreuve des bouleversements profonds de son environnement et préserver jusqu'à nos jours son identité (De Geus, 1997).

Cet article s'intéresse plus particulièrement à la pérennité organisationnelle. En effet, les autres formes de pérennité, tant en termes de contrôle et de pouvoir qu'en termes d'activités, ont fait l'objet de nombreuses études, faisant appel à des disciplines connexes (droit, fiscalité). En revanche, la pérennité organisationnelle est apparue plus récemment comme objet de recherche. Elle est apparemment plus complexe, bien que son étude se situe au cœur des sciences de gestion, entendues comme les disciplines traitant du pilotage des organisations. L'ambition du présent travail est d'apporter une contribution à un thème insuffisamment exploré alors que son enjeu nous semble fondamental ${ }^{2}$.

Nous avons choisi d'éclairer la pérennité de l'entreprise non pas par une énumération des stratégies «gagnantes» dans un cadre propre à «l'intention stratégique»- pour éviter le piège de dresser un «modèle unique» de cette pérennité -, mais en nous attardant au «processus stratégique» (Mintzberg, 1978;

1. Cette typologie est issue d'une étude lexicale de questions ouvertes contenues dans un questionnaire soumis à un échantillon d'entreprises pérennes.

2. À l'enjeu social et économique viennent s'ajouter les résultats des études récentes sur la durée de vie des entreprises. En effet, seule la moitié des firmes nouvellement créées en France sont toujours présentes après cinq ans d'existence (étude réalisée par l'INSEE en 1997 sur la création et le devenir des entreprises entre 1987 et 1995). Ces données sont confirmées sur le plan européen par le quatrième rapport mené par la Commission européenne (Projet PME, Eurostat 1996). Enfin, l'étude de De Rooij (1996), réalisée sur un échantillon mondial d'entreprises, souligne que l'espérance de vie d'une firme serait de 12,5 ans. La rareté des entreprises ayant su défier ces statistiques éveille une curiosité certaine et mérite l'intérêt du chercheur.

Revue internationale P.M.E., vol. 15, nº 2, 2002 
Schendel et Hofer, 1979; Pettigrew, 1985, 1987, 1992; Van de Ven, 1992 ; Chakravarthy et Doz, 1992), c'est-à-dire au processus de formulation des stratégies aboutissant à la pérennité de l'entreprise.

La forme prise par l'évolution des événements d'un processus constitue un élément crucial. Il en découle, en effet, plusieurs familles de théories explicatives des processus. Ainsi, Van de Ven (1992) distingue les théories de cycle de vie, selon lesquelles les trajectoires sont prédéterminées, les théories téléologiques, qui reposent au contraire sur l'hypothèse d'équifinalité (plusieurs chemins possibles pour arriver à un résultat), les théories dialectiques, qui postulent la recherche d'équilibre entre forces opposées et, enfin, les théories évolutionnistes, qui expliquent l'évolution par une relation récursive et cumulative entre variation, sélection et rétention.

Notre vision théorique pour cerner ce concept complexe qu'est la pérennité emprunte à la fois aux théories téléologiques, dans la mesure où nous mettons l'accent sur la volonté comme principe d'évolution, aux théories dialectiques, dans la mesure où changement et continuité sont bien deux forces qui s'opposent et dont la confrontation est source d'évolution ; enfin, elle se rattache aux théories évolutionnistes puisque l'évolution est aussi perçue comme une boucle entre variation, sélection et rétention.

Parmi ces différents courants, il s'agira de mettre en évidence la manière dont les contextes internes et externes (au sens de Pettigrew) modèlent l'évolution stratégique. Le cadre d'analyse obtenu est qualifié d' «approche intraorganisationnelle de l'équilibre ponctué »; « équilibre ponctué » parce qu'il s'intéresse à l'alternance dialectique entre deux forces contraires, les changements et la continuité rythmant la vie d'une entreprise ; « approche intraorganisationnelle » parce que nous adoptons non pas une vision déterministe ou de contingence externe pour expliquer les phases d'évolution de la firme, mais tentons en interne de mettre en évidence le processus de décision stratégique conduisant à ces périodes de stabilité ponctuées par des changements de grande ampleur.

Ce cadre permettra d'aborder, d'une part, les stratégies d'adaptation et, d'autre part, les stratégies de proaction. Le processus de pérennisation sera étudiée à partir des initiatives stratégiques d'une PME ayant fait l'objet d'une étude de cas approfondie ${ }^{3}$.

3. Les principales initiatives stratégiques prises depuis la création de l'entreprise (en 1760) jusqu'à nos jours ont été répertoriées. Neuf d'entre elles ont été analysées en profondeur (Mignon, 1998), cinq sont présentées dans le cadre de cet article.

Revue internationale P.M.E., vol. 15, $\mathrm{n}^{\circ}$ 2, 2002 
En effet, les avancées théoriques laissent certaines questions en suspens : quel est le contenu de ces contextes internes propres à l'adaptation et la proaction d'une entreprise pérenne ? Comment ces derniers se couplent-ils à des facteurs tels que le contexte externe, la volonté, l'expérimentation, le degré d'anticipation?

L'étude de cas vise à répondre à ces questions. Sans sous-estimer « la robustesse » des modèles initiaux, ces derniers doivent être enrichis et adaptés à partir des informations et enseignements issus de l'observation du terrain.

\section{Stratégies adaptatives}

Dans un premier temps sera étudiée l'adaptation de l'entreprise en tant que processus d'ajustement à son environnement. Parmi les nombreuses définitions données à son concept, nous avons opté pour celle de Danjou (1987) pour qui une adaptation est réalisée lorsque «l'entreprise a exercé la possibilité de choisir entre plusieurs alternatives distinctes dictées par les conditions passées et présentes » de son contexte interne et externe. Dans un deuxième temps, l'étude historique des initiatives stratégiques adaptatives d'une entreprise de plus de deux cents ans permettra de préciser le contenu des concepts retenus.

\subsection{Les avances théoriques}

Après avoir rappelé l'origine du cadre d'analyse retenu (dernières avancées de l'écologie intraorganisationnelle), la spécificité du processus d'adaptation d'une entreprise pérenne sera mise en évidence.

\subsubsection{L'intégration du courant écologique au champ du management stratégique}

La proposition centrale de ce dernier courant de recherche (Burgelman, 1991, 1996; Burgelman et Mittman, 1994 ; Barnett et Burgelman ; 1996 ; Montgomery, 1995 ; Levinthal et March, 1981) est que les stratégies des organisations sont déterminées à la fois par un processus de sélection interne et externe.

On retrouve le schéma général de «variation-sélection-rétention » propre à l'écologie des populations, mais cette fois transposé en interne. Les deux approches, malgré leurs similitudes, ne doivent pas être confondues, car elles reposent sur des méthodologies très différentes (quantitative vs qualitative) adaptées à des objectifs spécifiques (étude de populations d'organisations vs analyse du fonctionnement interne des organisations).

Revue internationale P.M.E., vol. 15, nº 2, 2002 
Dans le cadre de l'écologie intraorganisationnelle, les « variations ${ }^{4}$ » prennent la forme d'initiatives autonomes émanant le plus souvent de managers en contact direct avec les développements technologiques et les changements de condition de marché. La «sélection » se réalise à travers les mécanismes de gestion (systèmes de contrôle, règles gouvernant l'allocation des ressources) et les mécanismes culturels (rites, normes de conduite) assurant le couplage entre la stratégie organisationnelle et les initiatives des managers à différents niveaux hiérarchiques. L'efficacité des processus de sélection interne dépend de l'intensité avec laquelle ils reflètent les pressions de sélection externe (Burgelman, 1991, 1996). Certaines initiatives qui n'avaient pas été prévues ou planifiées peuvent ainsi devenir partie intégrante de la stratégie globale, lorsqu'elles ont démontré leur viabilité, eu égard à l'environnement de la firme. Chez Intel, par exemple, les activités EPROM et les microprocesseurs furent le fruit d'initiatives stratégiques qui n'avaient pas été prévues et planifiées et qui se situaient en dehors du champ stratégique de la firme au début des années 1970. La « rétention », enfin, prend la forme d'un apprentissage, engendrant la mise en place de routines, de croyances partagées assurant une stabilité à la stratégie sélectionnée.

La stratégie de l'organisation résulte ainsi en partie de la sélection et de la rétention des variations internes.

\subsubsection{Spécificité du processus d'adaptation des entreprises pérennes}

Une sélection réussie est à l'origine d'une période de stabilité (momentum) au sens de Tushman, Newman et Romanelli (1986) et Tushman et O'Reilly (1996). Noda et Bower (1996) expliquent, par exemple, que le choix des activités des firmes étudiées (deux Baby Bell : Bell South et US West) a été le résultat d'un processus concurrentiel entre diverses voies alternatives, mais qu'une fois le choix effectué, celui-ci a pesé sur le développement ultérieur des organisations. On voit donc ici l'apparition d'une certaine inertie qui rejoint bien la thèse du courant «écologie des populations », mais aussi les thèses des chercheurs en stratégie postulant que les firmes ayant réussi évoluent typiquement vers des «configurations » stables.

L'inertie se révèle cependant compatible avec des réajustements de grande ampleur, lorsque l'entreprise favorise le développement d'initiatives stratégiques variées, pour ensuite sélectionner les plus viables d'entre elles. Intel offre de nouveau l'exemple d'une firme dont l'inertie relative n'a pas empêchée de sortir à temps de l'activité DRAM.

4. Terme utilisé par les auteurs de ce courant (par analogie à l'écologie des populations) et qui signifie «changements » entrepris en interne par les cadres opérationnels.

Revue internationale P.M.E., vol. 15, nº 2, 2002 
À l'instar de Burgelman (1991), on peut dire que les entreprises pérennes sont caractérisées par l'équilibre entre une stratégie centralisée et des expérimentations de type de la base vers le haut laissant les mécanismes de sélection jouer. Enfin, les réorientations des entreprises pérennes sont plus souvent précédées de processus de sélection et d'expérimentation que les populations de firmes défaillantes.

La différence entre l'évolution d'une entreprise quelconque et une entreprise pérenne réside justement dans la qualité des filtres qui vont sélectionner les initiatives stratégiques. Les entreprises pérennes, en raison de leur ancienneté, présentent la particularité de devoir composer avec l'héritage de leur histoire passée, de leur culture, de leur savoir-faire issu des investissements matériels et immatériels. On peut donc penser que l'inertie présente chez ces entreprises les conduira à faire des choix dans la continuité des comportements porteurs de pérennité et donc à ne pas sélectionner des initiatives imprudentes, voire erronées.

\subsection{Interprétation empirique}

Cette étude empirique vise à spécifier les éléments du contexte interne dans le processus de naissance et de développement (ou de retrait) des initiatives stratégiques qui contribuent à l'évolution de l'entreprise. Elle tentera donc de décrire le contenu et les interrelations entre les deux «moteurs» de pérennité. Ce faisant, nous souhaitons mettre en évidence le rôle de l'intentionnalité, du volontarisme des dirigeants dans la conduite de leur entreprise vers la pérennité et la forme par laquelle elle s'exprime (expérimentation ou planification).

La méthodologie ${ }^{5}$ qui s'est imposée est de type qualitatif. La condensation, la présentation des données et l'élaboration/vérification des conclusions se sont inspirées de Yin (1994), Miles et Huberman (1991) et Eisenhardt (1989). Les données primaires ont été recueillies sous forme d'entretiens semi-directifs réalisés auprès des cadres et dirigeants de l'entreprise. Les données secondaires se composent pour l'essentiel de données internes à l'entreprise (documentation, archives).

La méthode employée dans ce contexte méthodologique est l'étude de cas (Hlady-Rispal, 2000) à l'instar de celle d'auteurs de l'écologie intraorganisationnelle ( $c f$. Burgelman, 1996, sur Intel ; Noda et Bower, 1996, sur deux Baby Bell : Bell South et US West). En effet, les auteurs fondateurs de ce courant s'intéressent au processus de formation des stratégies. L'approche ne doit pas être confondue avec celle bien connue de l'écologie des populations dont la problématique suppose une approche statistique sur de larges échantillons.

5. Le détail de la méthodologie, notamment concernant le codage des données et la présentation des résultats ainsi que la vérification de leur fiabilité et validité (Yin, 1989, 1994), est fourni dans Mignon (1998).

Revue internationale P.M.E., vol. 15, $\mathrm{n}^{\circ}$ 2, 2002 


\section{Présentation de l'entreprise 6}

Griset SA est une entreprise employant 243 personnes (CA en 1997 : 420 millions de francs), fondée en 1760 dans le quartier du Marais, dont les activités sont, d'une part, la fonderie et le laminage des métaux non ferreux (cuivre, laiton, bronze, aluminium et alliages) et, d'autre part, la construction de matériel de fonderie et de laminage (Griset Engineering) ${ }^{7}$.

Dans la perspective de l'équilibre ponctué, histoire de l'entreprise Griset fait apparaître quatre grandes phases ponctuées par des dates clés.

a) Une longue période de modernisation et de diversification, de 1750 à 1956, où la firme acquiert ses deux principaux métiers : la fonderie et le laminage de métaux non ferreux. C'est également pendant cette période qu'elle réalise une diversification vers des produits qui constituent aujourd'hui encore sa principale production (même si les proportions produites et les débouchés évolueront au cours du temps): le cuivre, le bronze, le laiton et l'aluminium laminé.

b) Une deuxième période, de 1957 à 1972, pendant laquelle Griset reconquiert son indépendance (à l'égard de son fournisseur), indépendance qu'elle avait perdue à la suite de l'abandon de son métier de fonderie en 1953. Une date tout à fait cruciale est la création de la CFFL (Compagnie française de fonderie et de laminage) en 1967 qui permettra de mener à bien le développement de l'entreprise dans les années à venir.

c) Griset entreprend, de 1973 à 1996, son expansion. L'accroissement considérable de la valeur ajoutée de ces produits lui permet de retrouver des marges plus confortables, de réaliser les investissements nécessaires à l'accroissement de la production; Griset saisit aussi les possibilités d'expansion à l'étranger grâce à son département d'ingénierie (100\% des ventes à l'export).

6. Une monographie plus complète est présentée dans Mignon (1998).

7. Griset est restée indépendante jusqu'en décembre 1997, date à laquelle elle fut vendue au groupe familial allemand Diehl. Notre période d'observation s'étalant de septembre 1997 à février 1998, l'analyse portera principalement sur la période antérieure à la date de cession. Nous envisagerons ultérieurement le devenir de l'entreprise après son changement de contrôle.

Revue internationale P.M.E., vol. 15, nº 2, 2002 
d) Enfin, la décision d'intégration au groupe Diehl, en décembre 1997 complète sa stratégie d'expansion, cette fois-ci, au sein d'un grand groupe avec des moyens financiers accrus, mais aussi avec les contraintes que cela implique.

Il apparaît à notre sens deux dates clés, constituant des « ruptures », des tournants décisifs au sens de l'équilibre ponctué :

- 1967, création de la CFFL, qui présentera un risque majeur pour l'entreprise mais qui sera aussi le gage de son développement et de sa pérennité.

- 1997, cession à Diehl dans la mesure où elle représente un abandon de la pérennité du pouvoir. De plus, si elle sauvegarde la pérennité organisationnelle, elle engendre des changements culturels avec l'adoption d'une stratégie beaucoup plus formalisée et anticipée qu'elle ne l'était auparavant.

\section{Le processus d'adaptation}

Nous avons choisi de détailler quatre processus, dont deux développements d'initiatives. 11 s'agit, d'une part, de la production de bandes double épaisseur (adaptation par l'offre de nouveaux produits), d'autre part, de Griset Engineering (adaptation par l'adjonction d'une nouvelle activité) et deux retraits d'initiatives stratégiques, les arrêts de la production de lames de jalousies et d'ailettes d'aluminium. Ces initiatives ont été synthétisées dans une matrice de dynamique de site (Miles et Huberman, 1991, p. 213) ${ }^{9}$.

Il convient aussi de préciser que les extraits des citations proviennent principalement d'entretiens avec les cadres dirigeants (PDG et DG); cela s'explique par le fait que le processus de décision stratégique dans cette PME reste très centralisé ${ }^{10}$.

8. On pourra se référer à Mignon (1998) pour comprendre que les critères de choix de Diehl correspondaient à une volonté de la part du dirigeant-propriétaire de sauvegarder les composantes essentielles de la pérennité organisationnelle de l'entreprise.

9.Signalons que les éléments de contexte interne font partie des éléments de continuité répertoriés dans cette étude de cas ( $c f$. Mignon). En outre, une étude complémentaire menée sur un échantillon de 30 entreprises pérennes a permis de mettre en évidence (à l'aide d'une analyse lexicale des réponses à des questions ouvertes) que la pérennité d'une entreprise découlait d'un équilibre entre éléments de changement et de continuité. Il apparaît, grâce à ce cas, que ces deux forces dialectiques sont aussi récursives : en effet, les éléments de continuité (l'histoire, les axes stratégiques, les valeurs, etc.), loin de s'opposer au changement, contribuent et orientent ce dernier dans le sens de la pérennité de l'organisation.

10. Les questions relatives aux facteurs de pérennité (exposées dans Mignon, 1998) ont en revanche reçu des réponses de la part de l'ensemble du personnel interrogé (soit une dizaine de cadres au total).

Revue internationale P.M.E., vol. 15, nº 2, 2002 


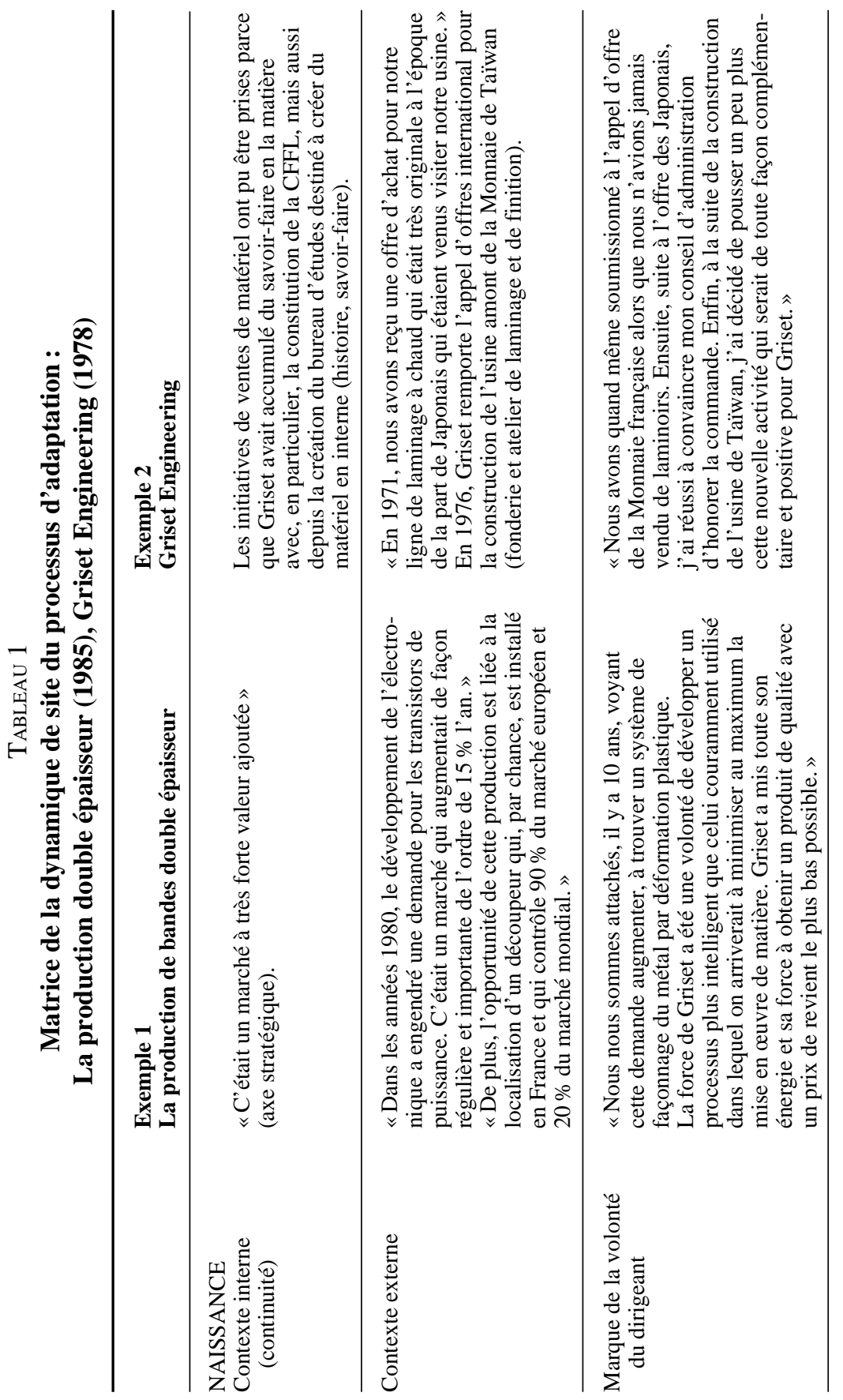

Revue internationale P.M.E., vol. 15, nº 2, 2002 


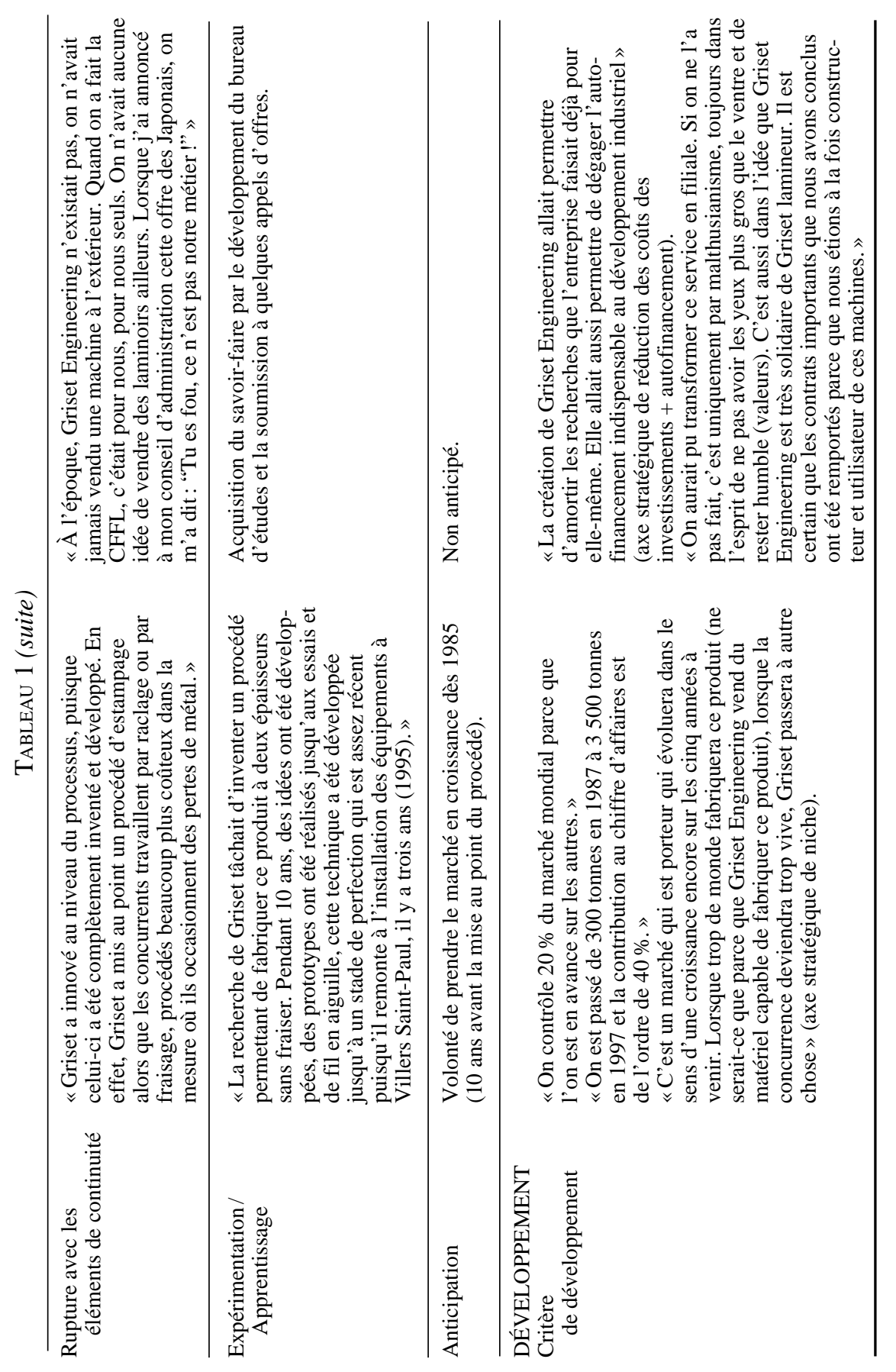

Revue internationale P.M.E., vol. 15, $\mathrm{n}^{\circ}$ 2, 2002 
La naissance et le développement d'une initiative stratégique en vue d'une adaptation sont donc clairement conditionnés par un contexte interne (axe stratégique, valeurs, culture, histoire) et un contexte externe (concurrence, demande).

Ces contextes internes, en particulier l'axe stratégique, centré sur la recherche d'amélioration des coûts et de la qualité, semblent même prévaloir sur le contexte externe. On trouve là l'argument central de l'écologie intraorganisationnelle : la sélection interne joue à travers les mécanismes administratifs et culturels régulant l'allocation des ressources, la clé étant que la sélection interne reflète en fait les pressions de la sélection externe (Barnett et Burgelman, 1996). Dans notre cas, c'est bien cette volonté de se développer conformément à un certain nombre de principes et conformément à un axe stratégique : focalisation sur une niche, produit technique, complexe et de qualité, choix guidés par la prudence et le pragmatisme, qui ont permis à l'entreprise de rester sur le marché. Il convient, à cette occasion, de souligner le caractère volontariste du processus d'adaptation. Cette constance dans la stratégie et les valeurs est, par ailleurs, explicitement reconnue comme un des facteurs de pérennité de l'entreprise.

L'histoire de l'entreprise, est-elle aussi fondamentale ? En effet, c'est riche de toute son expérience passée qu'une entreprise est capable d'intégrer dans son processus de décision les erreurs à ne plus commettre et les éléments de son passé auxquels elle souhaite rester fidèle. Si cette histoire peut être source d'inertie et à terme de sclérose, son rôle est plus ambigu qu'il n'y parait au premier abord, car elle peut aussi être facteur d'apprentissage et donc d'évolution. Les entretiens montrent, en effet, que le retour aux racines permet au dirigeant de prendre du recul et de prendre des décisions en harmonie avec un certain état d'esprit, une certaine ligne de conduite. L'histoire a donc été classée lors de l'analyse ( $c f$. matrices) parmi les éléments du contexte interne influant les décisions stratégiques de l'organisation étudiée.

Dans le passé d'une entreprise pérenne, il y a ainsi des constantes qu'elle cherchera à promouvoir si elles sont réellement explicatives de réussite ou avec lesquelles elle décidera de rompre si elles apparaissent comme des freins.

Une entreprise jeune jette donc les bases de sa pérennité future en développant les valeurs qui joueront par la suite le rôle de filtre aux décisions stratégiques.

Les expérimentations et l'apprentissage tiennent une place de choix dans le processus d'adaptation. La double épaisseur, par exemple, a abouti au bout de dix années de recherche, ce qui met, d'ailleurs, en exergue une valeur essentielle accompagnatrice du processus d'expérimentation : la persévérance.

Cette capacité d'adaptation a donc permis à Griset d'avancer, de se développer, tout en respectant une ligne de conduite forte qui l'a gardée de se fourvoyer.

On peut relever que les mécanismes poussant au développement stratégique sont les mêmes qui conduisent à son retrait.

Revue internationale P.M.E., vol. 15, nº 2, 2002 


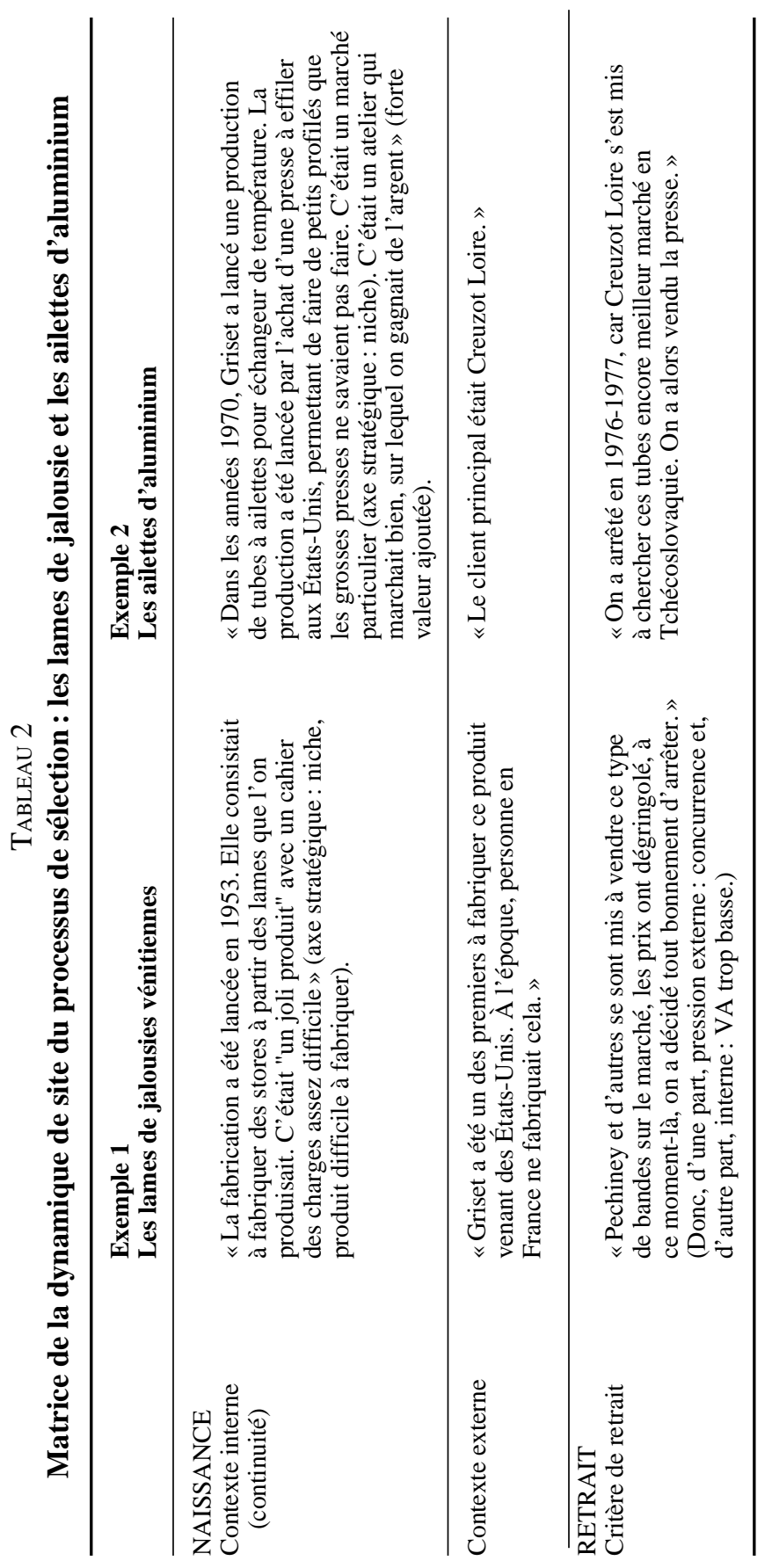

Revue internationale P.M.E., vol. 15, $\mathrm{n}^{\circ}$ 2, 2002 
On voit clairement que l'abandon des produits répond en effet à des pressions du marché (manque de rentabilité, concurrence trop vive), mais aussi, et surtout, à une rupture avec l'axe stratégique de la firme. À partir du moment où le produit devient un produit de masse sur lequel les fabricants se livrent à une guerre des prix, à partir du moment où la valeur ajoutée n'est plus importante et que le produit devient banal à fabriquer, il est abandonné. Ainsi, il s'agit bien des mêmes critères qui conduisent au lancement et au retrait des initiatives stratégiques.

Passons maintenant aux stratégies proactives de l'entreprise conduisant à une transformation de son organisation et/ou de son environnement à l'égard de ses clients ou de ses concurrents.

\section{Stratégies proactives}

Non seulement l'entreprise réagit par un processus d'adaptation, mais elle «proagit » par un processus de construction de son organisation et de son environnement (Koenig, 1996). L'attention sur ce dernier aspect de l'évolution des entreprises mettra l'accent sur deux points : l'objet de la transformation, c'est-à-dire le contexte interne et externe de l'entreprise et le mode de formulation de ce processus, et cela tant sur un plan théorique qu'empirique.

\subsection{Les points de vue théoriques}

Si l'adaptation découle du filtrage exercé par les contextes internes et externes, la proaction, au contraire, vise à agir sur ces contextes, à les transformer. Si l'adaptation se nourrit souvent de l'expérience passée de l'entreprise, la proaction constitue, quant à elle, une rupture par rapport à cet héritage.

\subsubsection{Le processus de construction organisationnelle et concurrentielle}

Selon les modèles soulignant le rôle proactif du manager, le destin des entreprises est avant tout entre les mains de ses dirigeants: «La stratégie d'une entreprise, son organisation, son environnement même sont modelés par les intentions et les actions des individus qui détiennent le pouvoir en son sein. » (Desreumaux, 1996, p. 99)

Le management d'une entreprise dans une logique proactive touche à la fois le contexte interne et externe de la firme.

Le mécanisme de transformation du contexte interne d'une organisation peut être étudié à la lumière des modèles d'apprentissage et des modèles de l'équilibre ponctué. En effet, l'apprentissage en « simple boucle » contribue à l'adaptation des 
théories ${ }^{11}$ utilisées «théorie par l'agir» aux théories affichées (espoused theory). Elle est source de continuité, de stabilité organisationnelle, appelées «phase de convergence », par les théoriciens de l'équilibre ponctué. Au contraire, les «phases de réorientations » sont caractérisées par une forte instabilité organisationnelle entraînant un apprentissage en « double boucle » ${ }^{12}$, une remise en cause des théories affichées et des théories utilisées. C'est lors de ces réorientations qu'intervient la remise en cause du contexte interne de l'entreprise. Les recherches sur le déclin montrent aussi que les changements de grande ampleur passent par une remise en cause des filtres cognitifs qui façonnent les structures organisationnelles (Nystrom et Starbuck, 1984). Modifier le contexte interne, c'est-à-dire le «paradigme » (Koenig, 1996) au sein duquel se développe la vision stratégique n'est certes pas chose aisée, tant celui-ci façonne les interprétations, routines et comportements des membres d'une organisation. Pourtant, c'est à ce prix qu' une entreprise peut changer le cours de sa trajectoire, la faire volontairement dévier de celle induite par son passé, son histoire ou par les contraintes de son environnement.

La question qui se pose est de savoir jusqu' où va la remise en cause des contextes internes d'une entreprise pérenne, réalisant un changement de type proactif. La littérature nous donne quelques éléments à ce sujet. Collins (1997) précise à cet égard que les changements de grande ampleur ne remettent pas en cause les valeurs centrales de l'entreprise. «Dans une entreprise vraiment performante dans la durée, la philosophie centrale perdure en tant que référence et source d'inspiration. Elle assure la cohésion d'une entreprise au cours de ses différentes phases de croissance, de décentralisation, de diversification, d'internalisation et de mutation interne $\gg($ p. 110).

Koenig (1996) rappelle à ce sujet le point de vue de Hedberg, Nystrom et Starbuck (1976) sur la pérennité d'une entreprise. Celle-ci découle de l'équilibre trouvé entre diverses exigences : équilibre entre consensus et dissension, équilibre entre satisfaction et sens critique, équilibre entre aisance et écoute de son environnement, équilibre entre capacité d'apprentissage et de désapprentissage.

Il semble donc qu'à travers tous ces équilibres, ce sont avant tout la juste mesure, le refus de l'excès et la modération qui sont recherchés, caractéristiques qui semblent fondamentalement représenter les entreprises pérennes. Un chef

11. Le terme théorie est utilisé dans un sens très spécial par Argyris et Schön (1978). Il s'agit de l'ensemble des règles structurant l'action individuelle et organisationnelle (Charue, 1991).

12. Argyris et Schön (1978) relèvent, en se fondant sur la typologie de Bateson (1977), plusieurs niveaux d'apprentissage : l' « apprentissage en simple boucle » consiste en des changements qui ne remettent pas en cause les principes de l'action organisationnelle, l' « apprentissage en double boucle » conduit à un changement des normes guidant l'action organisationnelle, le «double apprentissage» vise à une organisation apprenante. 
d'entreprise exposait, dans des termes semblables, sa vision de la pérennité : «Il s'agit de trouver un équilibre entre prudence et audace, valeurs traditionnelles et progrès, rigueur et humanisme.»

La proaction consiste aussi à transformer le contexte externe de l'entreprise. En effet, la stratégie ne doit pas se cantonner à une démarche d'adaptation à l'environnement, elle doit également contribuer à le construire.

L'exemple de Sony ayant littéralement créé la demande pour le walkman dans les années 1980 est un exemple de proaction. En effet, une entreprise n'a pas forcément besoin que son environnement change pour commencer à changer, elle peut anticiper des changements futurs ou encore les provoquer. Il ne faut donc pas sous-estimer les possibilités dont elle dispose pour «créer les opportunités... et façonner les menaces» (Koenig, 1996). Cette capacité de création et de transformation s'exprime tant à l'égard de la demande (création d'un produit, marché) qu'à l'égard de la concurrence. En effet, les coopérations interentreprises (alliances, réseaux), les concentrations sont bien la manifestation d'une volonté d'organiser, voire de «fermer» les marchés. La législation sur les ententes et abus de position dominante est la preuve que cette capacité de proaction est bien vivace au point qu'elle nécessite parfois d'être limitée.

\subsubsection{La formulation du processus}

Les théoriciens des ressources de base (Wernerfelt, 1995 ; Collis et Montgomery, 1995 ; Barney, 1991, 1996; Yeoh et Roth, 1999) sont à l'origine d'avancées significatives en matière de stratégie proactive. Le modèle de l'intention stratégique, élaboré par Hamel et Prahalad (1989), crée délibérément un écart entre les ressources et les ambitions dans une logique proactive alors que le modèle de l'adéquation consistait à adapter les ressources disponibles aux occasions d'affaires existantes (Allouche et Schmidt, 1995).

Lorino et Tarondeau (1998) proposent d'enrichir les théories des ressources de base par une vision de la stratégie fondée sur les processus. Ces ressources deviennent stratégiques «lorsqu' elles sont exploitées dans des processus qui remplissent les conditions nécessaires pour être qualifiées de stratégiques... c'est-à-dire, lorsque ces derniers modifient les conditions d'insertion de la firme dans son environnement et lui procurent des avantages concurrentiels durables » (p. 15).

Encourager les stratégies proactives, c'est favoriser le débat d'idées en vue de l'apprentissage. À cette fin, Nystrom et Starbuck (1984) préconisent de tenir compte des avis, événements, idées qui ne rentrent pas dans le cadre commun de l'organisation. Au sein de l'entreprise, la culture doit veiller à promouvoir l'esprit de contradiction, la possibilité de s'écarter des normes. Doivent être encouragés la 
curiosité, les points de vue divergents, l'écoute à des questions auxquelles on n'a pas de réponse immédiate, l'usage d'analogies et de métaphores (Hamel et Prahalad, 1995).

En effet, chaque expérimentation conduit à éclairer les zones d'ombre, à cerner la justesse de la stratégie, à la moduler pour qu'elle puisse satisfaire, in fine, les besoins des clients. Car, même dans le cas d'une stratégie proactive, le marché reste en dernier ressort le juge de l'initiative stratégique.

Une communication «au sens fort» (Giordano, 1997) doit être établie, c'est-à-dire une communication contribuant « au processus de co-construction du changement» (Giroux et Giordano, 1998, p. 139).

En résumé, il est souhaitable d'encourager l'expérimentation, source d'apprentissage, mais il faut également savoir provoquer des désapprentissages, nécessaires pour réaliser les ruptures conduisant aux réorientations stratégiques de l'entreprise. Argyris (1995) souligne le lien entre le processus de changement et le processus d'apprentissage. En effet, il est, d'une part, possible d' «apprendre à changer », mais en même temps, ce processus requiert un abandon des raisonnements et comportements habituels, il faut donc aussi « changer pour apprendre ».

\subsection{Mise en perspective empirique}

Nous souhaitons illustrer et enrichir les avancées théoriques précédemment exposées par l'étude d'une initiative ayant conduit à la proaction de la firme étudiée.

\subsubsection{Présentation du processus de proaction d'une entreprise pérenne}

L'exemple choisi est la création en 1967 de la CFFL (Compagnie française de fonderie et de laminage), car cette décision correspond à un véritable tournant stratégique au sens de l'équilibre ponctué. Quelles ont été les constantes qui ont permis la naissance puis le développement de cette initiative stratégique ? Comment s'est-elle couplée à d'autres facteurs tels que le contexte externe, la volonté, l'expérimentation, le degré d'anticipation ? Quels types de ruptures cette réorientation a-t-elle imposés? Les réponses à ces questions sont synthétisées dans une matrice de dynamique de site.

\subsubsection{Interprétation du processus de proaction d'une entreprise pérenne}

On voit clairement que les initiatives stratégiques de cette matrice aboutissent à la proaction, c'est-à-dire à une action de création, de transformation de l'organisation ou de l'environnement de l'entreprise. En effet, la création de la CFFL a pour

Revue internationale P.M.E., vol. 15, $\mathrm{n}^{\circ}$ 2, 2002 


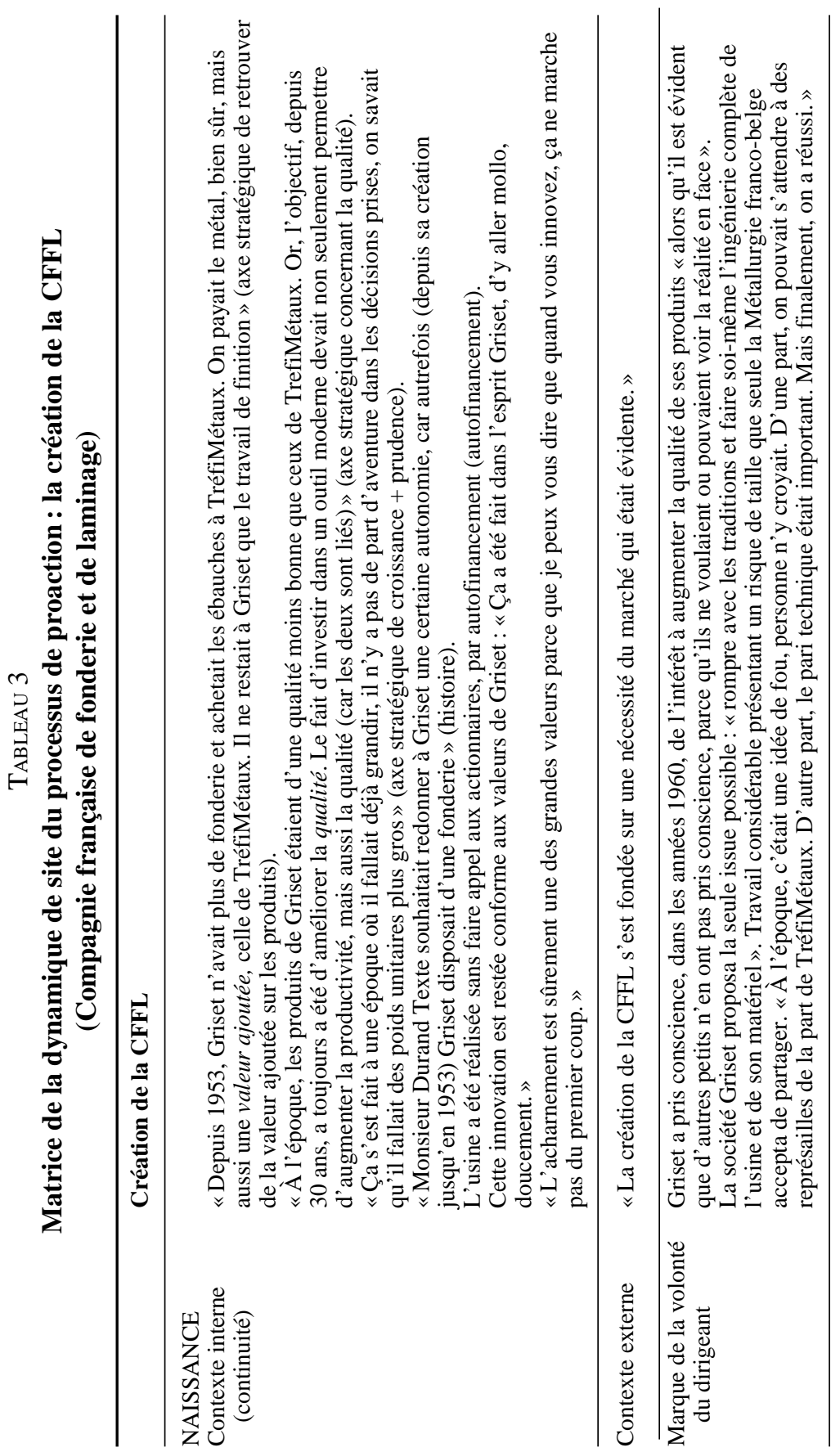

Revue internationale P.M.E., vol. 15, nº 2, 2002 


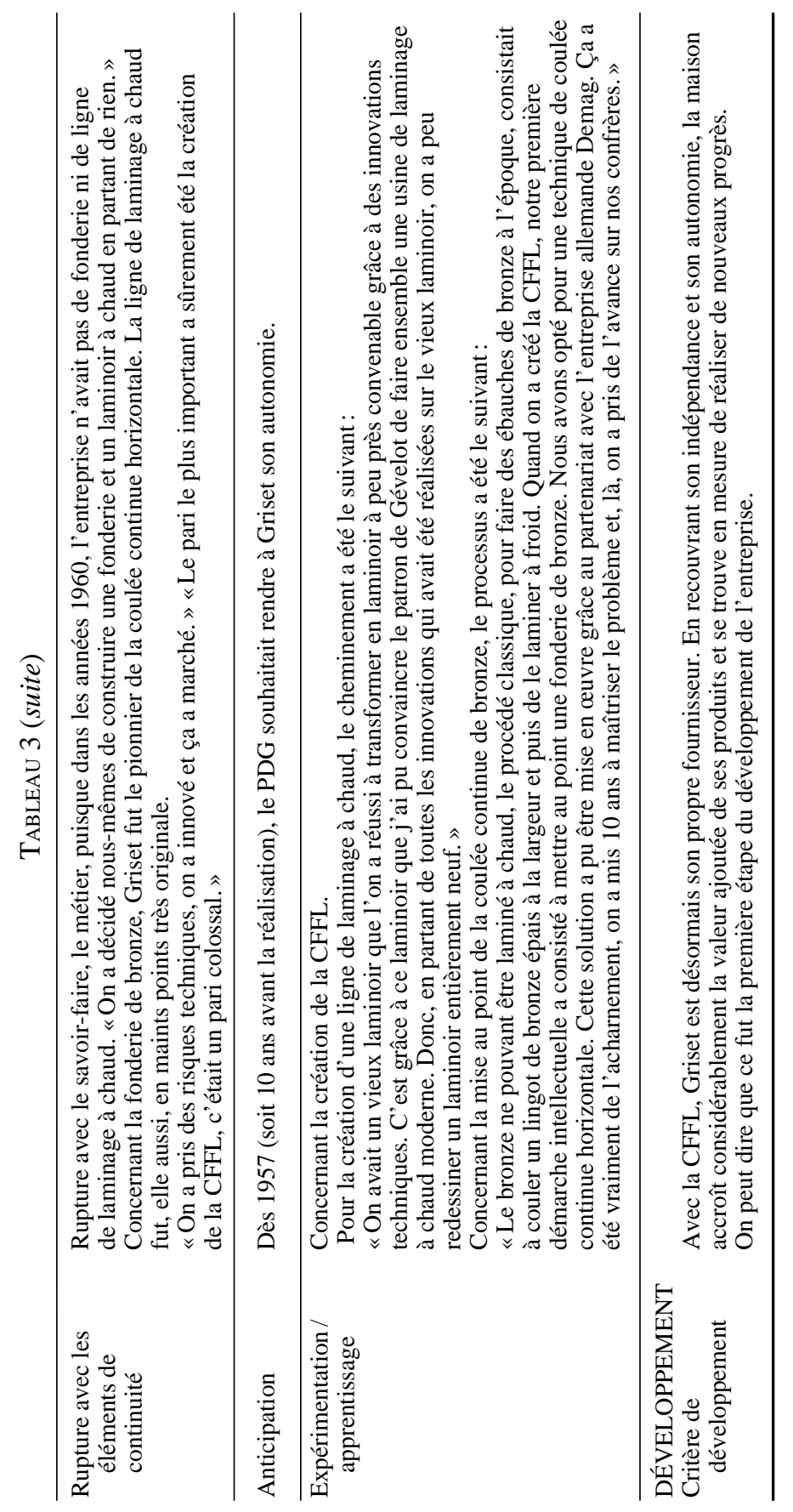

Revue internationale P.M.E., vol. 15, $\mathrm{n}^{\circ}$ 2, 2002 
conséquence l'indépendance de Griset par rapport à son fournisseur TréfiMétaux, leader du marché. Cette décision a constitué, aux dires des membres de la direction et des cadres de l'entreprise, le virage le plus important et le plus risqué de son histoire. Par cette décision, Griset se donne non seulement en interne les moyens de mettre en œuvre la stratégie désirée, mais elle transforme la configuration du marché et ses relations avec ses partenaires.

Même si cette décision est en congruence avec l'environnement de l'entreprise, elle représente un risque trop important pour que l'on puisse parler de seule adaptation, à tel point qu'elle a été perçue à l'intérieur comme à l'extérieur comme étant à contre-courant. On remarque aussi que, conformément à la relation entre «faible performance » et «pérennité » (Miller, 1994 ; Bolton, 1993), il semble que le potentiel de progrès en termes de qualité des produits ait impulsé la décision de s'affranchir de son fournisseur et de créer la CFFL.

Concernant les processus de formation de ces stratégies, si les initiatives stratégiques sont en rupture avec certains éléments de continuité du contexte interne comme le savoir-faire et le métier, elles sont cependant toujours guidées par les valeurs de l'entreprise comme la prudence, l'avancement pas-à-pas, le pragmatisme mais aussi la persévérance, voire l'acharnement, comme en témoigne l'importance des expérimentations qui ont permis à l'entreprise d'arriver à ses fins.

Nous rejoignons ici les thèses de Collins et Porras (1996) pour lesquels les valeurs constituent des éléments immuables, même si ces valeurs ne sont pas explicitées dans une charte comme dans les entreprises de leur échantillon. Dans notre étude, celles-ci sont tacites et perpétuées par l'éducation et la tradition. Ces valeurs constituent l'élément essentiel du contexte interne qui n'est pas sujet à des ruptures ni à des modifications; elles constituent l'élément de continuité par excellence dans la vie d'une entreprise pérenne.

\section{Conclusion}

La pérennité apparaît comme le résultat de trois volets d'évolution :

- l'abandon des initiatives stratégiques permet à l'entreprise de ne pas persévérer dans des voies irréalistes ;

- l'adaptation lui permet de ne pas être éliminée par son environnement lorsque ce dernier vient à changer ;

- la proaction lui permet de rebondir vers de nouveaux horizons et de ne pas faire de la pérennité une simple question de survie.

Les théories formant notre cadre d'analyse ont éclairé notre problématique à plus d'un titre. Elles ont montré, en effet, que l'adaptation et la proaction sont le 
fruit d'un mécanisme de sélection des initiatives stratégiques par des contextes internes et externes. Les stratégies pertinentes une fois retenues contribuent à des périodes de stabilité, d'inertie (convergent change) régulièrement remises en cause lors de changements de grande ampleur (upheaval change).

Le contenu des contextes internes et externes œuvrant à l'évolution d'une firme pérenne a été relevé à l'aide de l'étude empirique, en particulier les éléments du contexte interne propre à l'adaptation (axe stratégique, histoire, savoir-faire, valeurs) et les éléments propres à la proaction (prédominance des valeurs).

Cette étude a donc permis de mettre au jour le mécanisme par lequel l'entreprise placée devant plusieurs options stratégiques retient celle qui lui semble la plus pertinente en termes de pérennité : en effet, les « constantes » répertoriées sont autant de filtres, permettant de retenir ou au contraire d'abandonner une initiative stratégique. Les exemples à la fois de développement et d'abandon d'initiatives stratégiques ont montré que ces dernières sont gouvernées de façon symétrique par l'adéquation / inadéquation à l'égard de constantes identiques. Ces dernières, loin de s'opposer au changement, contribuent et orientent ce dernier dans le sens de la pérennité de l'organisation.

Il est clair que les résultats avancés sont contingents eu égard aux caractéristiques particulières de la firme étudiée : entreprise familiale de taille moyenne. En cela, l'étude se distingue des analyses classiques sur la pérennité organisationnelle qui portent en général sur des grandes entreprises cotées (De Geus, 1997; Collins et Porras, 1996). Alors que ces derniers associent la pérennité à une situation de leadership mondial, à une stratégie planifiée axée sur la croissance, la présente étude montre au contraire qu'une entreprise peut opter délibérément pour une taille moyenne et assurer sa pérennité par une stratégie de niche. Si cette constatation vient confirmer les thèses de Penrose (1959), elle infirme celles de Chandler (1991) qui, dans un article sur « les principes éternels du succès industriel », avance que «pour gagner, il faut jouer la carte de la grande taille».

Les caractéristiques de la stratégie de Griset sont typiquement celles d'une PME (Marchesnay, 1993 ; Marchesnay et Fourcade, 1997) : la prise de décision reste très centralisée, le projet stratégique est fortement imprégné des buts et valeurs du dirigeant, les rapports avec les différents partenaires sont très personnalisés. L'horizon de la stratégie est bien de long terme, mais le projet n'est pas formalisé, ce qui permet de promouvoir l'adaptabilité, le caractère évolutif et la flexibilité de l'entreprise. «Cette évolutivité est liée, non seulement aux péripéties et manœuvres mais également aux processus d'apprentissage, au travers d'essais et d'erreurs, de tâtonnements, mais aussi de l'acquisition progressive de savoir-faire, qui confèrent progressivement à l'entreprise sa compétence distinctive.» (Marchesnay et Fourcade, 1997, p. 83)

Revue internationale P.M.E., vol. 15, $\mathrm{n}^{\circ}$ 2, 2002 
Parmi les huit degrés qui caractérisent le mode de formation de la stratégie répertoriés par Mintzberg et Waters (1985) menant du volontarisme au déterminisme stratégique, celui de Griset peut être qualifié d'entrepreneurial (Julien et Marchesnay, 1996; Verstraete, 1999).

La stratégie prend source dans la vision cardinale d'un leader, mais cela n'implique pas que les différentes initiatives soient rigoureusement articulées les unes aux autres. La stratégie est en conséquence susceptible d'intégrer les opportunités qui se présentent. Placée sous le contrôle du chef d'entreprise, l'organisation vit à l'abri d'une niche. La stratégie est relativement délibérée mais peut intégrer des éléments émergents. (Cité par Koenig, 1996, p. 35.)

Cette vision stratégique du dirigeant (Filion, 1991; Verstraete, 1999; Calori, Very et Arregle, 1997 ; Nkongolo-Bakenda, d'Amboise et Garner, 1994) imprègne fortement le processus de formation des stratégies de pérennisation.

Le dirigeant de la PME pérenne contribue fortement à l'équilibre requis pour perdurer. L'équilibre s'opère en premier lieu entre son intelligence conceptuelle et son intelligence pratique. S'il se doit d'avoir une vision sur le long terme, un bon manager se reconnaît également à sa capacité à être sur le terrain (compétences techniques) et surtout à savoir fédérer le personnel autour d'un projet commun. Le chef d'entreprise, par sa formation, sa maîtrise de soi, exerce souvent un pouvoir charismatique sur les membres de son entreprise. Ses qualités de rigueur se tiennent en équilibre avec une volonté profonde d'équité et de compréhension à l'égard du personnel. Enfin, l'atteinte de cette pérennité constitue une sorte de victoire sur le temps et sur lui-même.

La présente étude illustre ainsi à sa manière l'une des multiples facettes « des réalités de l'entrepreneuriat» (Verstraete, 2000). Cette recherche confirme également les thèses relatives aux caractéristiques propres aux entreprises familiales - stratégie financière axée sur la prudence (Allouche et Amann, 2000 ; Mahérault, 1998; Gallo et Vilaseca, 1996 ; Hirigoyen, 1984), horizon de long terme (Allouche et Amann, 2000 ; Harvey, 1999), management stratégique imprégné de valeurs (Hau, 1995) et vient compléter les nombreux travaux de recherche sur la stratégie des entreprises familiales ( $c f$. aussi les états de l'art réalisés par Wortman, 1994, Allouche et Amann, 2000 ; Ward, 1987 ; Donckels et Frölich, 1991 ; Daily et Dollinger, 1992 ; Daily et Thomson, 1994; Gudmundson, Hartman et Tower, 1999; Habbershon et Williams, 1999; Mouline, 2000). En effet, si la plupart d'entre eux étudient l'influence de l'actionnariat sur le comportement stratégique des firmes familiales ou le délicat problème de la succession du contrôle et de la direction, peu abordent le problème du processus de formation des stratégies. Ce champ de recherche largement exploré pour des grandes entreprises cotées nous a amenée à transposer les concepts au cas d'une PME pérenne. L'objectif était de renforcer la validité interne et externe de la recherche au sens de Eisenhardt (1989) et de tendre vers 
une généralisation analytique au sens de Yin (1994) par le rapprochement de résultats similaires obtenus dans des contextes différents (en termes de taille, d'âge et de contrôle).

Néanmoins, il est évident que cette recherche mériterait d'être approfondie. Il est en effet souhaitable qu'une multiplication des sites étudiés puisse confirmer ou infirmer les résultats obtenus et montrer une nouvelle fois l'intérêt d'approfondir le champ spécifique des entreprises entrepreneuriales et familiales.

\section{Bibliographie}

Allouche, J. et B. AmAnn (2000), «L'entreprise familiale : un état de l'art », Finance Contrôle Stratégie, vol. 3, $\mathrm{n}^{\circ} 1$, p. 33-80.

Allouche, J. et G. SCHMIDT (1995), Les outils de la décision stratégique, tome 2, Paris, La Découverte.

ARGYRIS, C. (1995), Savoir pour agir: Surmonter les obstacles à l'apprentissage organisationnel, Paris, InterÉditions ; Éditions Dunod en 2000.

ARGYRIS, C. et D. SCHÖN (1978), Organizational Learning : A Theory of Action Perspective, Reading, Mass., Addison-Wesley.

BARNETT, W.P. et R. BURGELMAN (1996), «Evolutionary perspectives on strategy », Strategic Management Journal, vol. 17, p. 5-21.

BARNEY, J. (1991), «The resource-based model of the firm: origins, implications and prospects », Journal of Management, vol. 17, $\mathrm{n}^{\circ} 1$, mars.

BARNEY, J. (1996), «The resource-based theory of the firm », Organization Science, vol. $7, \mathrm{n}^{\circ}$ 5, septembre-octobre, p. 469.

BAteson, G. (1977), Vers l'écologie de l'esprit, Paris, Seuil.

Bolton, M.K. (1993), «Organizational innovation and substandard performance: When is necessity the mother of innovation?», Organization Science, vol. 4, février, p. $57-75$.

BURGELMAN, R.A. (1991), «Intraorganization ecology of strategy making and organizational adaptation: theory and field research », Organization Science, vol. $3, n^{\circ} 2$, p. 139-261.

BURGELMAN, R.A. (1996), «A process model of strategic business exit : implications for an evolutionary perspective on strategy », Strategic Management Journal, vol. 17, p. 193-214.

BURGELMAN, R.A. et B.S. MitTMAN (1994), «An intraorganizational ecological perspective on managerial risk behavior, performance, and survival : individual, organizational and environmental effects », dans J.A.C. Baum et J.V. Singh (dir.), Evolutionary Dynamics of Organizations, New York, Oxford University Press, p. 53-75.

CAlori, T., P. Very et J.L. ArRegle (1997), «Les PMI face à la planification stratégique », Revue française de gestion, $\mathrm{n}^{\circ}$ 112, janvier-février, p. 11-23.

CHAKRAVARTHY, B.S. et Y. Doz (1992), «Strategy process research », Strategic Management Journal, vol. 13.

Revue internationale P.M.E., vol. 15, $\mathrm{n}^{\circ}$ 2, 2002 
Chandler, A.D. (1991), «Les principes éternels du succès industriel», Harvard L'Expansion, hiver, p. 82-92.

CHARUE, F, (1991), Apprentissages organisationnels et mutation industrielle : l'exemple de la robotisation des tôleries automobiles, Thèse de doctorat de l'École des mines de Paris.

CoLlins, J.C. (1997), « Le secret des entreprises qui durent », L'Essentiel du Management, janvier, p. 108-114.

COLLINS, J.C. et J.I. PORRAS (1996), Bâties pour durer, les entreprises visionnaires ontelles un secret?, Paris, First-Management.

Collis, D.J. et C.A. Montgomery (1995), «Competing on resources, strategy in the 1990s », Harvard Business Review, p. 118-143.

DAILY, C.M. et S.S. THOMSON (1994), «Ownership structure, strategic posture, and firm growth : an empirical examination », Family Business Review, vol. 7, n 3, p. 237-249.

DAILY, C.M. et M.J. Dollinger (1992), « An empirical examination of ownership, structure in family and professionally managed firms », Family Business Review, vol. 5, $\mathrm{n}^{\mathrm{o}} 2$, p. 117-136.

DANJOU, I. (1987), L'évolution des firmes : Analyse des facteurs et processus d'évolution à l'aide d'une approche monographique, Thèse de doctorat, Université de Lille I.

DE Geus, A. (1997), La pérennité des entreprises, Paris, Maxime Laurent du Mesnil.

DE RooIJ, E. (1996), A Brief Desk Research into the Average Life Expectancy of Companies in a Number of Countries, Stratix Consulting Group, août.

DESREumauX, A. (1996), « Nouvelles formes d'organisation et évolution de l'entreprise », Revue française de gestion, janvier février, p. 86-108.

DONCKELS, R. et E. FRÖLICH (1991), «Are family businesses really different ? European experiences from STRATOS », Family Business Review, vol. 4, n 2, p. 149-160.

EISENHARDT, K.M. (1989), «Building theories from case study research », Academy of Management Review, vol. 14, no 4, p. 532-550.

FILION, L.J. (1991), Visions et relations : clefs de succès de l'entrepreneur, Montréal, Les Éditions de l'Entrepreneur.

Gallo, M. et A. Vilaseca (1996), «Finance in family business », Familiy Business Review, vol. 9, $\mathrm{n}^{\circ} 4$.

GiordANO, Y. (1997), «L'action stratégique en milieu complexe: quelle communication?», dans M.J. Avenier (dir.), La stratégie «chemin faisant», Paris, Economica, p. 96-136.

GirouX, N. et Y. GiORDANO (1998), «Les deux conceptions de la communication du changement. », Revue française de gestion, septembre-octobre, p. 139-161.

Gudmundson, D., E.A. Hartman et C.B. Tower (1999), «Strategic orientation: differences between family and nonfamily firms », Family Business Review, vol. XII, $\mathrm{n}^{\circ} 1$, p. 27-39.

HABBERSHON, T.G. et M.L. WiLliAMs (1999), «A resource-based framework for assessing the strategic advantages of family firms », Family Business Review, vol. XII, n 1 , p. 1-26.

Revue internationale P.M.E., vol. 15, n 2, 2002 
HAMEl, G. et C.K. Prahalad (1989), «Strategic intent», Harvard Business Review, mai-juin, p. 63-76.

HAMEl, G. et C.K. Prahalad (1995), La conquête du futur, Paris, InterÉditions.

HARVEY, S.J. (1999), «Owner as manager, extended horizons and the family firm », International Journal of the Economis of Business, février, vol. 6, n 1, p. 41-55.

HAU, M. (1995), «Traditions comportementales et capitalisme dynastique : le cas des grandes familles », Entreprises et Histoire, n ${ }^{\circ}$ 9, p. 43-61.

Hedberg, B.L., P.C. Nystrom et W.H. Starbuck (1976), «Camping on seesaws : prescriptions for a self-designing organization », Administrative Science Quarterly, vol. 21, mars, p. 41-65.

HiRIGOYEN, G. (1984), Contribution à la connaissance des comportements financiers des moyennes entreprises industrielles et financières, Thèse de doctorat d'État, Université de Bordeaux I.

HLADY-RISPAL, M. (2000), «Une stratégie de recherche en gestion : l'étude de cas », Revue française de gestion, p. 61-70.

Julien, P.-A. et M. MARCheSnAy (1996), L'entrepreneuriat, Paris, Economica.

KoENIG, G. (1996), Management stratégique : paradoxe, interactions et apprentissages, Paris, Nathan.

LEVINTHAL, D. et J.G. MARCH (1981), «A model of adaptative organizational search », Journal of Economic Behavior and Organization, $\mathrm{n}^{\mathrm{o}} 2$ 2, p. 307-333.

LORINO, P. et J.C. TARONDEAU (1998), «De la stratégie aux processus stratégiques », Revue française de gestion, $\mathrm{n}^{\circ} 117$, p. 5-17.

MAHÉRAULT, L. (1998), «Des caractéristiques financières spécifiques aux entreprises familiales non cotées », Revue du financier, $\mathrm{n}^{\circ} 114$, p. 59-75.

MARChESNAY, M. (1993), «PME, stratégie et recherche», Revue française de gestion, $\mathrm{n}^{\circ}$ 95, septembre-octobre.

Marchesnay, M. et C. Fourcade (1997), Gestion de la PME/PMI, Paris, Nathan.

Mignon, S. (1998), Une approche de la pérennité de l'entreprise, Thèse de doctorat, Université de Nice Sophia-Antipolis.

Mignon, S. (2000), «La pérennité des entreprises familiales : un modèle alternatif à la création de valeur pour l'actionnaire ? », Finance Contrôle Stratégie, vol. 3, n ${ }^{\circ}$, p. $169-196$.

Mignon, S. (2001), Stratégie de pérennité d'entreprise, Paris, Vuibert.

Miles, B.M. et A.M. Huberman (1991), Analyse des données qualitatives, Bruxelles, De Boeck-Wesmael.

MiLlER, D. (1994), «What happens after success : the perils of excellence », Journal of Management Studies, vol. 14, p. 253-280.

MinTZBERG, H. (1978), «Patterns in strategy formation», Management Science, $\mathrm{n}^{\circ} 24$, p. 934-948.

MintZBERG, H. et J.A. WATERS (1985), « Of strategies, deliberate or emergent », Strategic Management Journal, vol. 6, n 257-272.

Revue internationale P.M.E., vol. 15, $\mathrm{n}^{\circ}$ 2, 2002 
MONTGOMERY, C.A. (dir.) (1995), Resource-Based and Evolutionary Theories of the Firm: Towards a Synthesis, Boston, Harvard Business School, Kluwer Academic Publisher.

Mouline, J.-P. (2000), «Dynamique de la succession managériale dans la PME familiale non cotée », Family Business Review, p. 197-222.

NKONGOLO-BAKENDA, J.-M., G. D’AMBOISE et B. GARNER (1994), «L'association entre la manifestation de la vision stratégiques des propriétaires-dirigeants de PME et la performance de leur entreprise », Revue internationale PME, vol. 7, p. 35-63.

NODA, T. et J.L. BowER (1996), «Strategy making as iterated processes of resource allocation », Strategic Management Journal, vol. 17, p. 159-192.

NySTROM, P.C. et W.H. STARBUCK (1984), « To avoid organizational crises : unlearn », Organizational Dynamics, printemps, p. 323-332.

Penrose, E. (1959), The Theory of Growth of the Firm, Oxford, Basil Blackwell.

PetTigrew, A.M. (1985), The Awakening Giant: Continuity and Change in ICI, Oxford, Basil Blackwell.

PetTigrew, A.M. (1987), «Context and action in the transformation of the firm », Journal of Management Studies, p. 633-670.

PetTigrew, A.M. (1992), « The character and significance of strategy process research », Strategic Management Journal, vol. 13, numéro spécial, p. 5-16.

SCHENDEL, D.E. et C.W. Hofer (dir.) (1979), Strategic Management, Boston, Mass., Little, Brown.

TUSHMAN, M.L. et C.A. O'REILly (1996), « The ambidextrous organization : management evolutionary and revolutionary change », California Management Review, vol. 38, $\mathrm{n}^{\mathrm{o}} 4$, p. 8-31.

Tushman, M.L., W.H. Newman et E. Romanelli (1986), « Convergence and upheaval : managing the unsteady pace of organizational evolution », California Management Review, vol. 29, n 1 , p. 29-44.

VAN DE VEN, A.H. (1992), «Suggestions for studying strategy process : a research note», Strategic Management Journal, vol. 13, numéro spécial, p. 169-188.

VERSTRAETE, T. (1999), Entrepreneuriat : connaître l'entrepreneur, comprendre ses actes, Paris, L'Harmattan.

Verstraete, T. (2000), Histoire d'entreprendre : les réalités de l'entrepreneuriat, Caen, EMS.

WARD, J.L. (1987), Keeping the Family Business Healthy: How to Plan for Continuing Growth, Profitability, and Family Leadership, San Francisco, Jossey-Bass.

WERNERFELT, B. (1995), «The resource-based view of the firm: ten years later », Strategic Management Journal, vol. 16, nº 3, p. 171-174.

WORTMAN, M.S. JR. (1994), «Theoretical foundations for family-owned business : a conceptual and research-based paradigm », Family Business Review, vol. 7, p. 3-27.

YEOH, P-L. et K. ROTH (1999), «An empirical analysis of sustained advantage in the U.S. pharmaceutical industry: impact of firm resources and capabilities », Strategic Management Journal, vol. 20, no 7, p. 637-653.

YIN, R.K. (1989, 1994), Case Study Research - Design and Methods, Londres, Sage Publications.

Revue internationale P.M.E., vol. 15, nº 2, 2002 